\title{
Adaptive Image Quality Enhancement with Hybrid Pixel Enhancement Approach
}

\author{
Sukhwinder Singh \\ Research Scholar (ECE) \\ SBSSTC, Ferozepur-152001, \\ Punjab, India
}

\author{
Amit Grover \\ Assistant Professor (ECE) \\ SBSSTC, Ferpozepur-152001, \\ Punjab, India
}

\begin{abstract}
Restoration is procedure of recoupling a picture from corrupted state. The image enhancement and restoration requires the image matrix processing, which utilizes the noise removal and contrast enhancement. The image enhancement method in the proposed model usually utilize the color enhancement, histogram equalization, color illumination, neighbor based reference model, non-reference image matrix enhancement and co-variance based matrix enhancement. In this paper, the combination of the neighbor based reference model and non-reference image matrix enhancement is proposed for the enhancement of the results. The experimental results have been performed over the grayscale standard images of the Lena, Baboon, Barbara and Peppers.
\end{abstract}

\section{Keywords}

Image enhancement, contrast enhancement, noise elimination, contrast adjustment.

\section{INTRODUCTION}

Image enhancement means to enhance the quality of the picture which the detail of image becomes more visible and clear [1]. It is vital task which can be used in several applications involving the digital video, World Wide Web, scientific imaging and DVDs. When large pixels inserted in the picture in order to enhance the size of picture and the main task is an enhancement of newest pixels from their surrounding real pixels [2]. It usually required a modification in picture dimensions given by non-inter factor, like as a $50 \%$ zoom where dimensions can be 1.5 times than real. There are several reasons to be executed to resize an image retaining as most as possible of information it contains. Image enhancement is useful in their different areas $[3,4]$. With the size of user based digital photographic, users can expect to take much control over digital images. Moreover, digital enhancement plays a role to pick up the cluesand descriptions in surveillance video and images. As HD television technology come the market place, and then many engineers take interest in large enhancement algorithmic programs for view traditional lowest definition programs on High Definition Television. Astronomical images take from probes and rovers which have received an extreme lowest transmission rate, to make transmission with high resolution of data infeasible [5, 6]. In the field of medical imaging, the neurologists have an ability to zoom theirParticular parts ofbrain tomography images.A similarApplication has been occurred when you want to increase their resolution of image while improving it using digital imaging software [7].To shorten the response time of browsing such web pages, images are often shown in low resolution forms. An enhanced higher resolution image is only shown if user clicks

on the corresponding thumbnailHowever, this approach still needs the image with high resolution to be stored on theserver and downloaded to the user's client machine on demand [8] To save storage space and communication bandwidth it would be desirable if the low resolution image is downloaded and then enhanced on the user's machine $[9,10]$.

\section{LITERATURE SURVEY}

Tzikas, D.G.[10] et al has worked on a new Bayesian model for the blind image deconvolution (BID) problem. In the proposed approach, a robust model of the BID preserves edges of the reconstructed image. Sparseness, robustness, and preservation of edges are achieved by using priors that are based on the Student's-t probability density function (PDF).Zhang X. F et al [11] has implemented the regularized anisotropic diffusion filter. In this paper, a regularized anisotropic diffusion filter was presented and USED to restore the DWI. The presented filtering strategy used for god preservation of edges. Rician noise, the PSNR and MOSIM metrics were used for measurement. Better results were found than conventional filters. Mateos, J. et al [12] has worked with new Bayesian methodology for the restoration of blurred and noisy images. Bayesian methods based on encapsulating prior image knowledge of image and avoid the ill-pawedness of image restoration problems. They use a spatially varying image prior utilizing a gamma-normal hyper prior distribution on the local precision parameters. The proposed restoration technique is better than other image.

\section{PROPOSED METHODLOGY}

Image acquisition is the initiative of the proposed model; image are loaded in to the memory of the image restoration model. When getting the image, consecutive step are to perform the low-rank recovery to restore the image. The go decomposition or wavelet decomposition technique will be used to produce the cube of original image and its decomposed matrices. Afterwards, the image would bear the pixel level restoration using the Non-documented restoration. Used technique can recover the missing element regions and can recreate the missing pixels. Then the image super element enhancement method are used to recover the pixel growth drawback occurred because of the image de-noising and restoration processes. Then, numerous performance parameters would be obtained within the form of mean square error (MSE) and peak signal to planned model are totally studied so as to conclude the findings and also the comparison of the planned and existing models are performed to gauge the development within the performance of the planned model

\subsection{Low-Rank matrix recovery model}

The LRMR technique compares the image matrix with the approximation matrix of the image to seek out the irregularity within the image knowledge. The irregularity matrix is termed the error matrix and subtracted from the first image to revive the image. During this paper, we tend to propose associate degree HSI restoration technique on the premise of low-rank 
matrix recovery (LRMR), which can simultaneously remove Gaussian noise, impulse noise, dead pixels or lines, and stripes. The main contributions of this paper are the exploration of the HSI low-rank property and the application of LRMR to the HSI restoration process.

\section{Algorithm 1: Low Rank Matrix Recovery}

i. At first, compute the N-level wavelet form decomposed after the wavelet decomposition method.

ii. The low rank recovery model formulates the parametric distribution based upon the statistical measurement of the above N-level wavelet coefficients, and the model parameters of the low rank model are estimated using the new technique.

iii. The distribution of the specified high-pass filtered ripple constant within the 0th level sub-band is calculable and so generates the 0th level ripple coefficients.

iv. Perform iterative operation on all input image pixels.

a. Compute the pixel rank and count the column space of current pixel.

b. Computer standard deviation for group of pixels on the basis of neighboring pixels.

c. Assign new rank to the current pixel.

v. Reassemble the matrix pixels to form the image matrix data

vi. Return the reconstructed matrix data.

\subsection{Multi-dimensional Non reference restoration pixel enhancement Algorithm}

Due to the various degrees of gap overlapping, two or more auxiliary pictures are required during a multi-temporal recovery procedure. It's typically the case that the obtainable pictures aren't adequate to fill all the missing pixels as a result of the gaps can't be fully covered. In this case, it's necessary to recover the remaining pixels employing a non-reference recovery technique. In this paper, a regularization method is presented for this problem. The recovery framework can be written as:

$p=\arg \min [E(p)]$

Where,

$$
E(p)=\left\|Q\left(p^{\prime}-p\right)\right\|^{2}+\lambda R(p)
$$

Is the cost procedure, during which $\mathrm{p}^{\prime}$ and $\mathrm{p}$ represent the input primary image and also the desired primary image, severally, $\mathrm{R}(\mathrm{p})$ represents the regularized model, and $\lambda$ is the regularization parameter. Here, $Q$ is a square matrix representing the reliable data within the different pixel locations, and its row number equals the image size. For the recovery issue, the component values are set to at least one for all the valid pixels, and to zero for the missing pixels. As for $\mathrm{R}(\mathrm{p})$, models like the Laplacian prior. By any considering the machine efficiency, the Laplacian model is more applicable for this work. Therefore, the cost function can be written as:

$E(p)=\left\|Q\left(p^{\prime}-p\right)\right\|^{2}+L \lambda p^{2}$

Where $\mathrm{L}$ is the Laplacian operator. The only parameter requiring predetermination in this method is the regularization parameter $\lambda$. According to Eq. (4.5), the regularization parameter $\lambda$ balances the info fidelity (the first term) and also the image regularization (the second term). In this paper, $\lambda$ was empirically set as 0.01 .

Differentiating E (p) with respect to $p$ gives:

$\nabla \mathrm{E}(\mathrm{p})=-2 \mathrm{Q}^{\mathrm{T}}\left(\mathrm{p}^{\prime}-\mathrm{p}\right)+2 L^{T} p$

Due to the problems involved with finding a solution to an equation like Eq. (4.6), an iterative technique using an initial guess to come up with successive approximations to a solution is usually adopted thus, the desired image can be solved by employing the successive approximations iteration:

$$
p_{n+1}=p n-\beta_{n} \nabla E\left(p_{n}\right)
$$

Where $n$ is the iteration range, and $\beta n$ is the step size. If $\beta n$ is simply too small, the convergence are very slow. However, if it's too large, the algorithmic rule are unstable or divergent. to deal with this issue, the conjugate gradient methodology is utilized in this paper. The step size can be calculated by:

$\beta n=\frac{\nabla^{2} E\left(p_{n}\right) p_{n}-Q^{T} Q p^{\prime} \nabla E\left(p_{n}\right)}{\left(\nabla E\left(p_{n}\right)\right)-\left(\nabla^{2} E\left(p_{n}\right)\right) \nabla E\left(p_{n}\right)}(6)$

Where $72 \mathrm{E}(\mathrm{p})$ is the Hessian matrix of the cost function $\mathrm{E}(\mathrm{p})$

\section{RESULTS ANALYSIS}

The very first challenge was to evaluate the performance of enhancement methods available for our research. For evaluated the performance of enhancement methods over the different kinds of images. The performance evaluation survey has helped to mark the difference between all of the shortlisted enhancement methods in order to obtain the best out of the given list. The results have been obtained in the form of resulting images and the performance parameters of image quality in the form of similarity and dissimilarity.

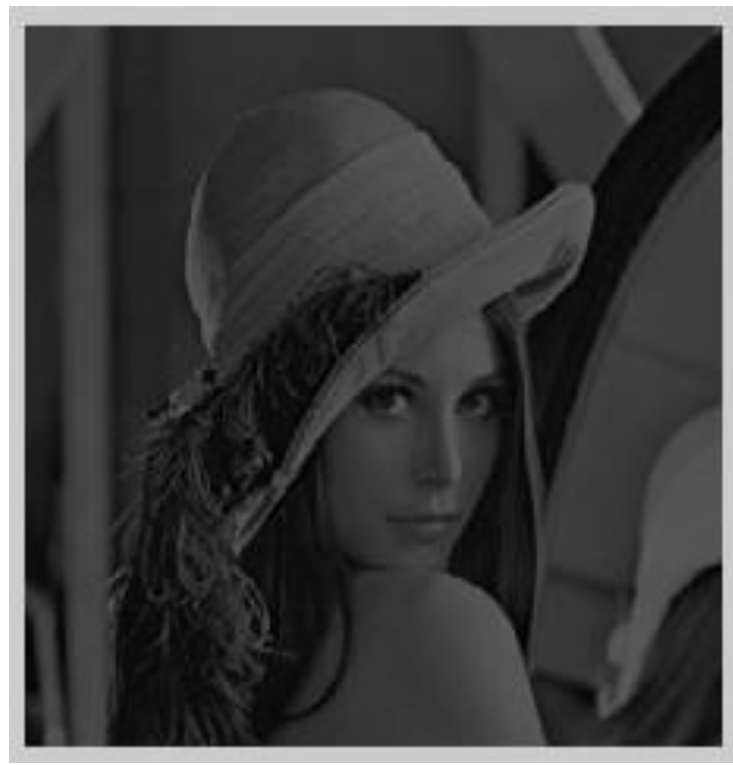

Figure1: Original Lena picture

The figure 1 shows the image before the contrast enhancement which shows the darker image, this picture has low quality because of darkness. 


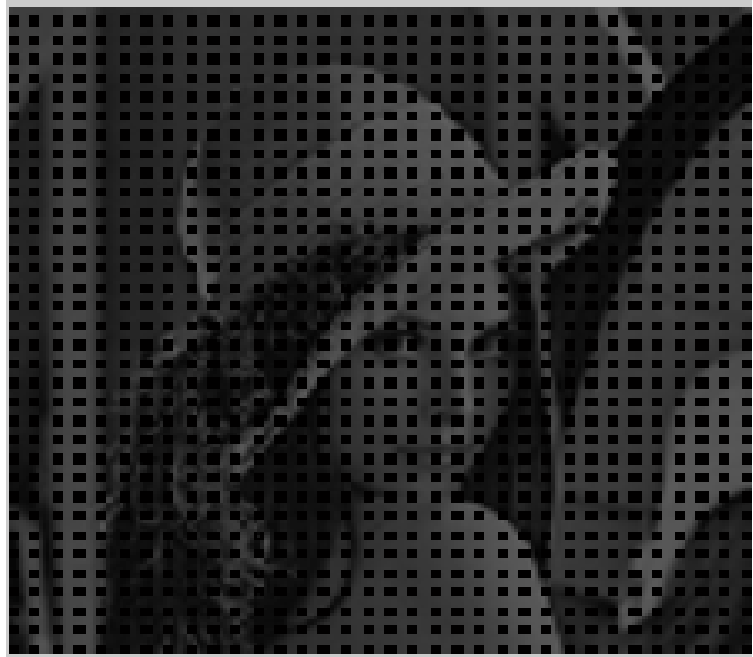

Figure 2: Image Enhancement results using LRMR.

The image enhancement results have been evaluated and tested with various image enhancement techniques. The image (Figure 2) shows the results obtained after the wavelet based enhancement method.

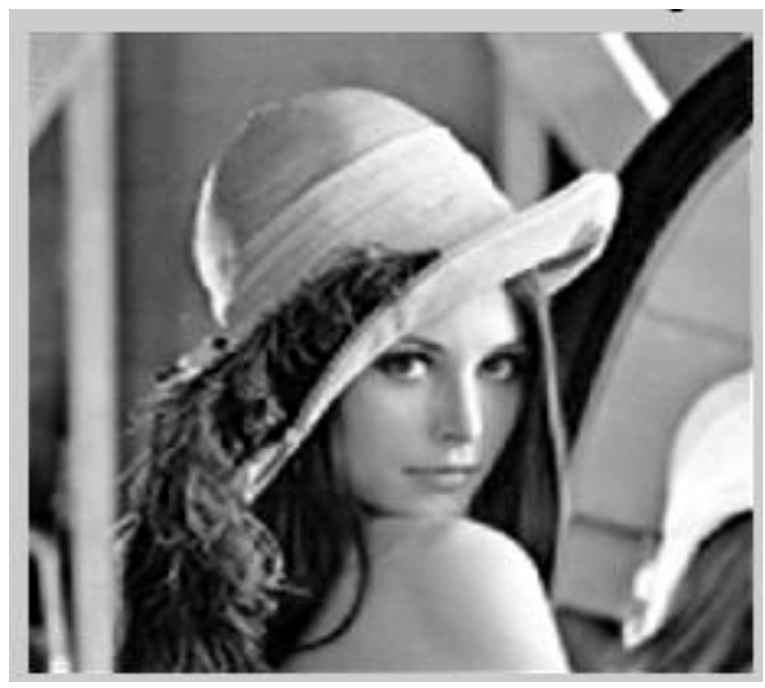

Figure 3: Image enhancement result obtained using the non-reference image matrix enhancement (Combined LRMR\&NRRA).

The image (figure 3 ) has been obtained after the application of proposed solution over the input Lena image. The Lena image is considered on the standard dataset images. The above image shows the results of low rank matrix recovery with the non-reference pixel enhancement method. The proposed model is based upon the co-variant enhancement method adds the low visibility squares over the input Lena image.

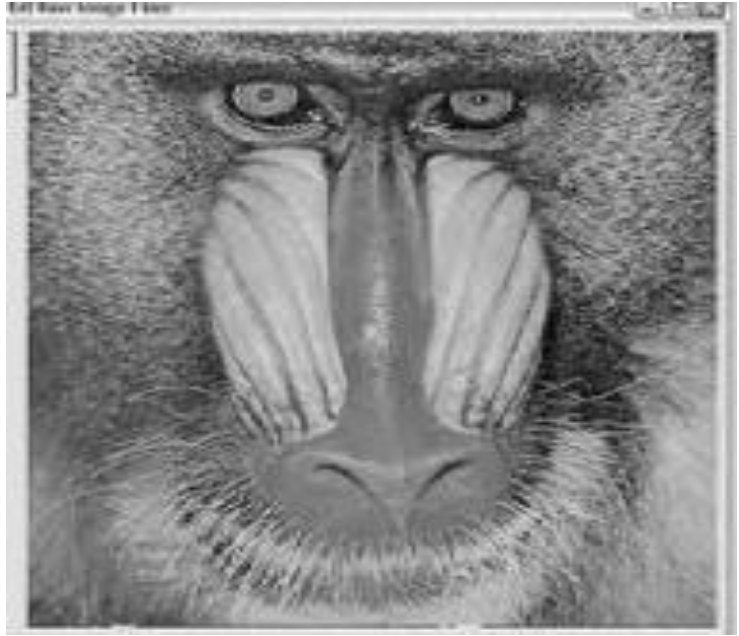

Figure 4: Original Baboon picture

The figure 4 shows the original Baboon picture. This image is without contrast enhancement.

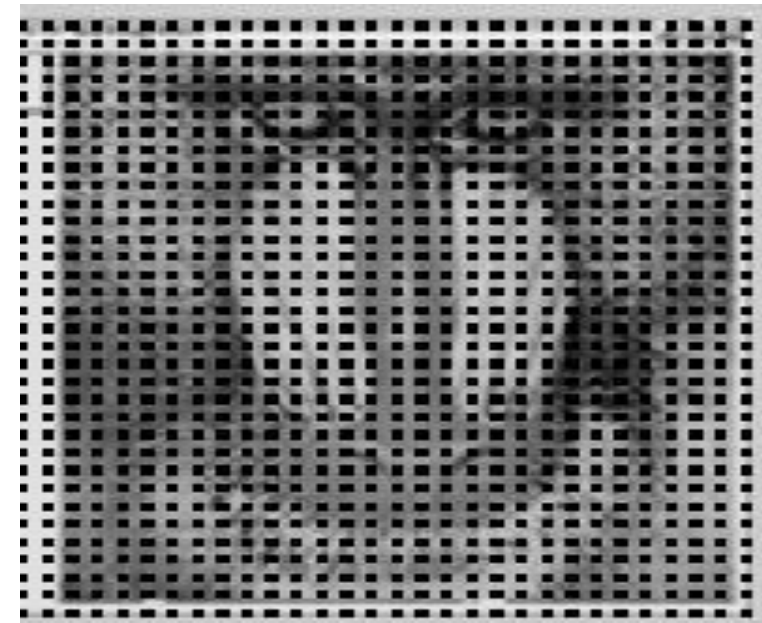

Figure 5: Image Enhancement results using LRMR.

The image in figure 5 has been achieved after the wavelet based enhancement technique.

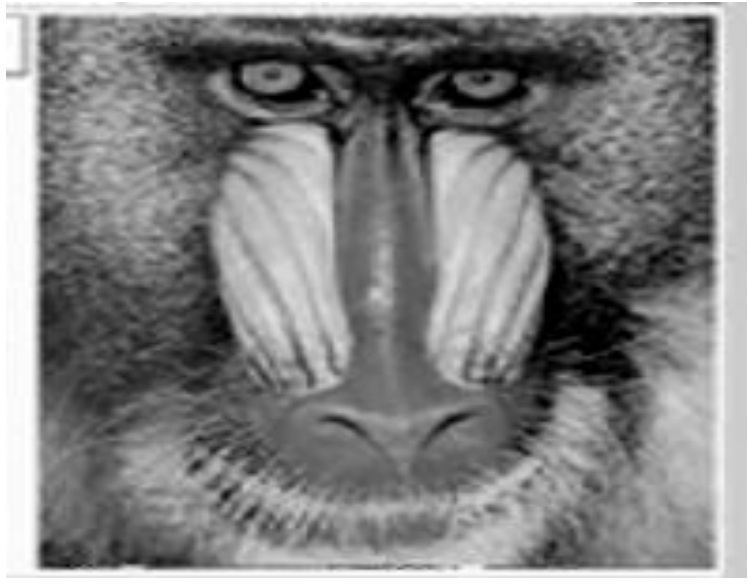

Figure 6: Image enhancement result obtained using the non-reference image matrix enhancement (Combined LRMR\&NRRA 
The above image (Figure 6) shows the results of low rank matrix recovery with the non-reference pixel enhancement method. The proposed model is based upon the co-variant enhancement method adds the low visibility squares over the input Baboon image. Figure 6 image is combined image of LRMR \& NRRAs

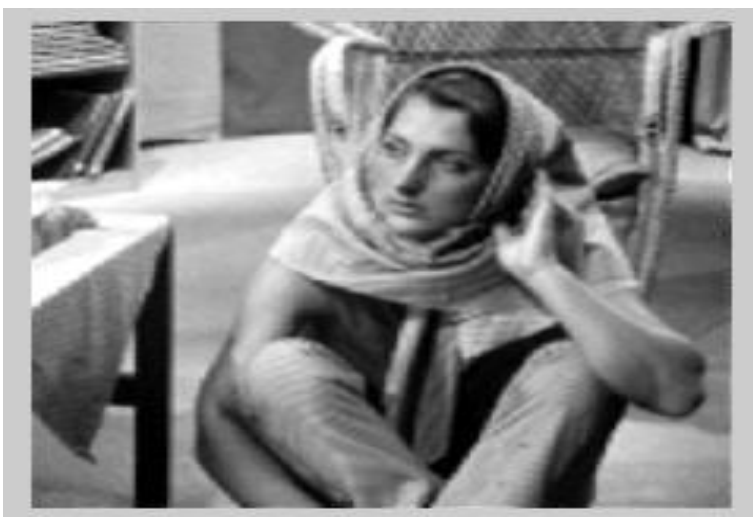

Figure 7: Original Barbara picture

Figure 7 consist of original Barbara picture which has low contrast as compare to NRRA method.

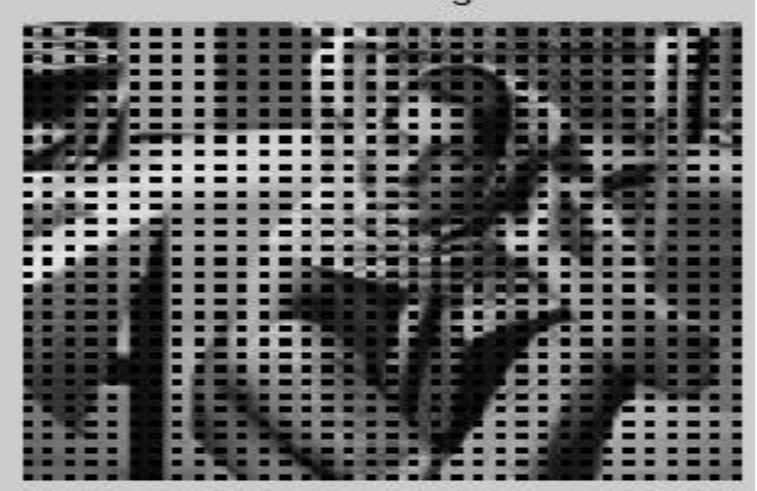

Figure 8: Image Enhancement results using LRMR

The image (Figure 8) shows the results obtained after the wavelet based enhancement method.LRMR technique shows the pixel in image.

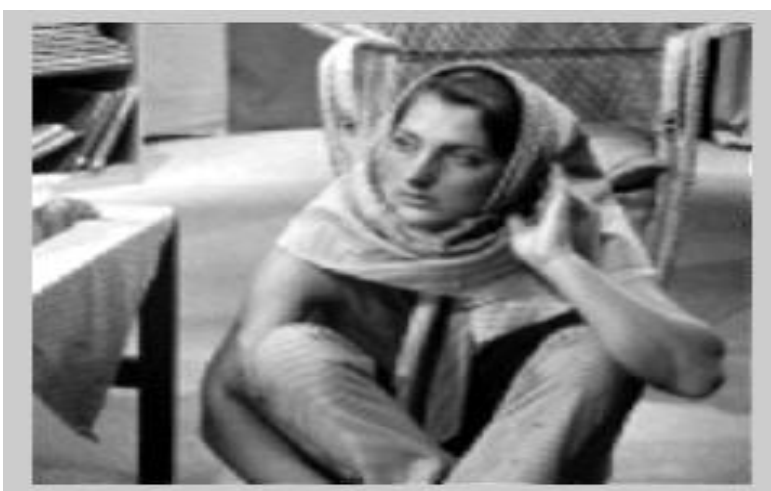

Figure 9 combined LRMR\&NRRA image

The image (figure 9) consist of both LRMR and NRRA method .This image has good contrast and quality as compare to original image and LRMR based image. The above image (Figure 9) shows the results of low rank matrix recovery with the non-reference pixel enhancement method.

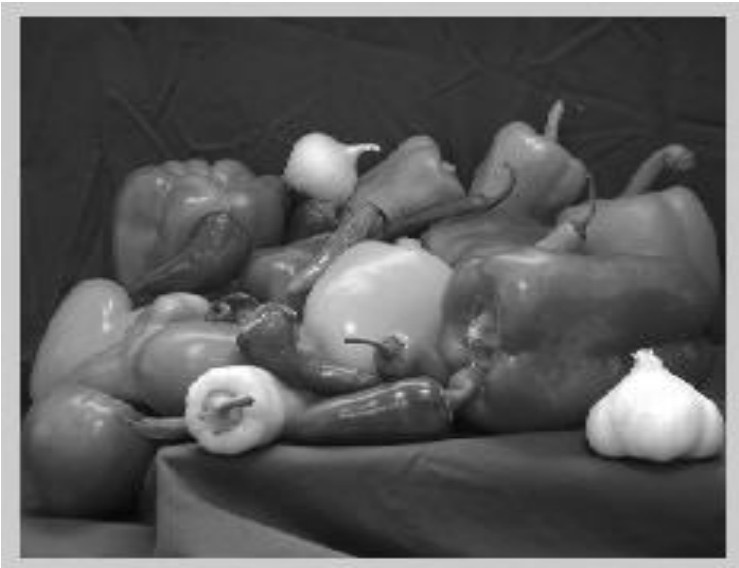

Figure 10: Original peppers picture

The image in Figure 10 consist of original peppers picture.

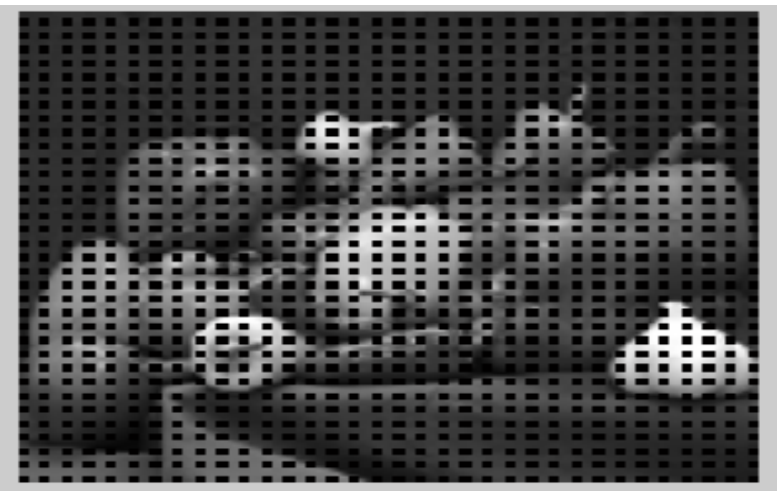

Figure 11: Image Enhancement results using LRMR

Figure 11 show the pixel in image. The LRMR method used as a pixel in matrix form in the image.

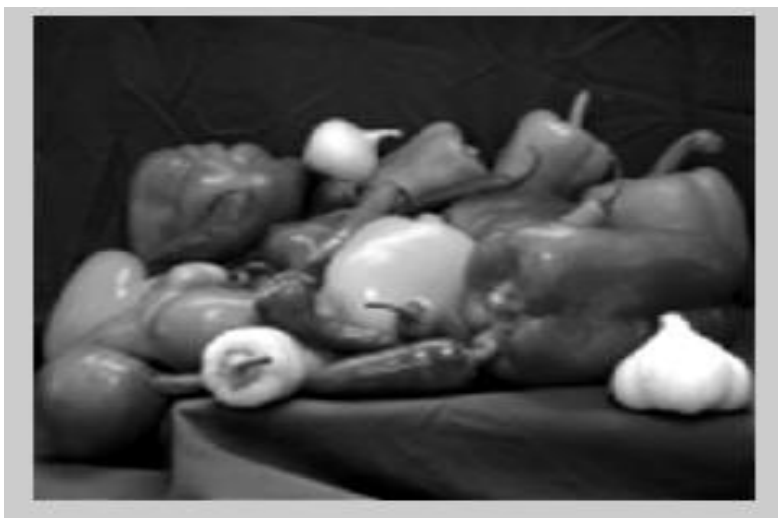

Figure 12: Combined LRMR\&NRRA image

The figure 12 consist of both LRMR and NRRA method .This image has good contrast and quality as compare to original image and LRMR based image. The above image (Figure 12) shows the results of low rank matrix recovery with the nonreference pixel enhancement method.

In this research work PSNR and MSE are used to measure the quality of an image. Peak signal to noise ratio (PSNR) and Mean square error (MSE) have been implemented in order to obtain some quantitative results for comparison of wavelet based enhancement techniques. It is cleared from table values that the new and adaptive image enhancement method is giving far better results than any other technique. Hence, this 
combined method has been recommended over the existing models.

\section{RESULTS}

The results obtained from the standard images have been evaluated in terms of PSNR and MSE in table 1.

Table 1. The evolution of standard image dataset from proposed model

\begin{tabular}{|c|c|c|c|c|}
\hline Object & Lena & Baboon & Barbara & Peppers \\
\hline PSNR & 21.002032 & 11.4784 & 13.5072 & 16.0244 \\
\hline MSE & 0.9995 & 0.9999 & 0.9999 & 0.9998 \\
\hline
\end{tabular}

Table 1 defines the performance measures in the form of PSNR and MSE after the application of the proposed method over the standard images of Lena, Baboon, Barbara and Peppers.

\section{CONCLUSION}

The proposed model is the amalgamation of the major image quality enhancement or restoration methods of non-reference pixel enhancement and matrix regularization method and lowrank matrix recovery. The proposed model has been tested with several prominent images which includes the Lena, Baboon, Barbara, and Peppers images. The results have obtained in the form of peak signal to noise ratio and mean square error. It has been cleared from table 1 that theproposed methodology is giving far better results than any other technique.The results shows that the proposed model outperforms the existing models when evaluated on the basis of peak signal to noise ratio and mean square error

\section{REFERENCES}

[1] A.Gotchev, J. Vesma, T. Saramaki and K. Egiazarian," Digital image re sampling by modified B-spline functions,"IEEE Nordic Signal Processing symposium, pp. 259-262, June 2000.

[2] A. M. Darwish, M. S. Bedair and S.I. Shaheen, " Adaptive resampling algorithm for image enhancement", IEEE Proc. Vis. Image Signal Process, vol. 144, No. 4, August 1997.
[3] AnderaGiachetti and Nicola Asuni, " Real-Time artifact free Image up scaling", IEEE Transactions on Image Processing, vol. 20 No. 10, October 2011.

[4] B. S. Morse and D. Schwartzwald,"Image magnification using level-set reconstruction", Proc. IEEE Int. Cof. Computer Vision Pattern Recognition, vol.3, pp. 333340, 2001.

[5] Buades, Antoni, BartomeuColl, and Jean-Michel Morel "Nonlocal image and movie denoising." International journal of computer vision 76.2, pp. 123-139, 2008

[6] C. B. Atkins, C. A. Bouman and J. P. Allebach, "Optimal image scaling using pixel classification”, Proc. IEEE Int. Conf. Image Processing, vol. 3, pp. 864-867, 2001.

[7] Concus, P., Golub, G. H., \& O'Leary, D. P. A generalized conjugate gradient method for the numerical: Solution of elliptic partial differential equations: Computer Science Department, School of Humanities and Sciences. : Stanford University, 1976

[8] Gonzalez, Rafael C. "RE woods, Digital Image Processing." Addison-Wesely Publishing Company (1992).

[9] H.chen and G. E. Ford,"An FIR enhancement filter Design method based on properties of HumanVision", Proc. IEEE Int. Conf. Image Processing, vol.3, PP.581585, November 1994.

[10] H.Jiang and C. Moloney,"A new direction adaptive scheme for image enhancement", Proc. IEEE Int. Conf. Image Processing, vol.3, pp.369-372, 2002. ) Tzikas, Dimitris G., Aristidis C. Likas, and Nickolaos P. Galatsanos. "The variational approximation for Bayesian inference." Signal Processing Magazine, IEEE 25.6 (2008): 131-146

[11] Zhang X. F, Ye H, Tian W.F, Chen W.F, "Denoising DWI Based on Regularized Filter," IEEE, pp. 120 121,10-11march 2007.

[12] Mateos, J., Bishop, T.E., Molina, R., Katsaggelos, A.K.,"Local Bayesian image restoration using variational methods and Gamma-Normal distributions," IEEE, Image Processing (ICIP), pp.129, 132, 7-10, nov2009. 